\title{
Discovered Solar Positronium
}

\author{
Nagendra Nath Mondal1,2 \\ ${ }^{1}$ Department of Physics, Batanagar Institute of Engineering, Management and Science (Techno India Group), \\ Kolkata, India \\ ${ }^{2}$ Department of Physics, Indian Institute of Engineering, Science \& Technology (IIEST), Shibpur, \\ Howrah, India \\ Email: nn.mondal2011@gmail.com
}

Received 20 September 2014; revised 20 October 2014; accepted 15 November 2014

Academic Editor: Luigi Maxmilian Caligiuri, University of Calabria, Italy

Copyright ( 2014 by author and Scientific Research Publishing Inc.

This work is licensed under the Creative Commons Attribution International License (CC BY).

http://creativecommons.org/licenses/by/4.0/

(c) (i) Open Access

\begin{abstract}
I describe a method for the observation of Positronium (Ps) involvement in the solar radiation spectrum. In this method, Rydberg-Ritz's principle and Planck's radiation formula are used to acquire information of the atomic transitions of Ps alike Hydrogen and Helium. In order to perform this experiment, an advanced solar spectrum monitor is constructed by utilizing light emitting diodes (LED) of various colors. A detailed study on this method provides qualitative agreement with experimental data, giving insight to the physical process involved in the solar radiation spectrum and confirming the existence of solar Ps.
\end{abstract}

\section{Keywords}

LED, Light, Positronium, Simulation, Spectrometer

\section{Introduction}

Living creatures can sustain only in the third planet of the solar system and maximum energies consumed by it are being received from the Sun. Positronium (Ps) is a purely leptonic H-like atom formed from an electron $\left(\mathrm{e}^{-}\right)$and its anti particle the positron $\left(\mathrm{e}^{+}\right)$. Particle antiparticle interaction is an electromagnetic process and a good test of QED leading to discover the similar phenomena in the environment of Sun by studying the chromaticity of the solar spectrum [1]. It is thought that solar energy produced by the radiation consisting of atomic transitions of $\mathrm{H}$ and $\mathrm{He}$, and what maximum of us did not aware of Ps which is one of the important ingredient of solar radiation. From the best of my knowledge on the experimental and the theoretical literature survey, solar Ps spectrum is being demonstrated here for the first time. Radioactive isotope, pair production and fusion reac- 
tion are the major sources of $\mathrm{e}^{+}$. The huge applications for $\mathrm{e}^{+}$include atomic, nuclear, astrophysics experiments [1]-[5], positron emission tomography (PET) [6] [7], studies of defects, surfaces, electron momentum of materials [8] [9] and material with medicinal values [10]. With the advent of sophisticated technology scientists are capable to produce high intense pulsed positron beam, accumulation of $\mathrm{e}^{+}$and Ps, production of $\mathrm{Ps}_{2}$ molecule, intensive studies of Ps laser cooling for the achievement of Ps Bose-Einstein Condensation (PsBEC) [11]-[15]. Short pulses of Ps atoms is suitable for laser spectroscopy of the Lyman- $\alpha$-like transition in the dipositronium $\left(\mathrm{Ps}_{2}\right)$ molecule at a UV wavelength of $251 \mathrm{~nm}$ [16], as well as Ps formation and dynamics in various target materials and efficient production of Rydberg Ps (binding energy $-6.8 \mathrm{eV}$ which is just half of the $\mathrm{H}$ due to the reduced mass of Ps) atoms. A. P. Mills and co-workers succeeded to make the $\mathrm{e}^{+}$beam intensity $\sim 10^{10}$ to $\sim 10^{11}$ per $\mathrm{cm}^{2}$ by adding Ps-forming target and a pulsed magnet and Ps-Lyman- $\alpha$ spectroscopy can be found elsewhere [17]. Dipositronium and Ps can be produced simultaneously on the metal surface by the highest intense slow $\mathrm{e}^{+}$beam bombardment which is significant for studying the Rydberg Ps atoms and observing the PsBEC state. Hence laboratory based $\mathrm{e}^{+}$and Ps studies enrich our knowledge that helps me to unearth the solar Ps and find out its characteristics in the enormous temperature of the Sun. The detection of Ps outside the laboratory experiments were first observed by Chuup et al. (1973) [18] and first identified by Levethal et al. (1978) from the Galactic centre [19] via detection of the Ps annihilation, but innovation of the solar light color spectroscopy did not exist. Recent novelty of light emitting diodes (LED) for which "Nobel Prize-2014" is conferred to Isamu Akasaki, Hiroshi Amano and Shuji Nakamura for their greatest contribution in social, industrial and entertainment applications. How LEDs can play a crucial role in science \& technology development and pave the way of discovering the solar Ps will be illustrated in the following sections.

\section{Theoretical aspect of Solar Positronium}

\subsection{Formation of Solar Positronium}

The fusion reaction [1] is a source of solar Ps production that persistent in thermal plasma at $T \leq 10^{5} \mathrm{~K} \quad$ [20] for a while that is good enough to measure the transition energies between the quantum states. Since the reduced mass of Ps is $m_{e} / 2$ just half of that of $\mathrm{H}$ atom and hence the binding energy of Rydberg Ps is $-6.8 \mathrm{eV}$. Correspondingly Ps-Lyman- $\alpha$ has a wavelength of $243 \mathrm{~nm}$ and was observed by Canter et al. [21]. The total wave function of the Ps state is the product of three wave functions depending on the spin, space and charge coordinates:

$$
\varphi(\text { total })=\varphi(\text { space }) \alpha(\text { spin }) \beta(\text { charge })
$$

This is a symmetric-antisymmetric wave function depends on the spin functions for a combination of two spin-1/2 particle-antiparticle ( $\mathrm{e}^{+}$and $\mathrm{e}^{-}$) can be expressed in the following four possible blends [22]:

$$
\begin{aligned}
& \alpha(1,1)=\varphi_{1}\left(\frac{1}{2}, \frac{1}{2}\right) \varphi_{2}\left(\frac{1}{2}, \frac{1}{2}\right), \\
& \alpha(1,0)=\frac{1}{\sqrt{2}}\left[\varphi_{1}\left(\frac{1}{2}, \frac{1}{2}\right) \varphi_{2}\left(\frac{1}{2},-\frac{1}{2}\right)+\varphi_{2}\left(\frac{1}{2}, \frac{1}{2}\right) \varphi_{1}\left(\frac{1}{2},-\frac{1}{2}\right)\right], \\
& \alpha(1,-1)=\varphi_{1}\left(\frac{1}{2},-\frac{1}{2}\right) \varphi_{2}\left(\frac{1}{2},-\frac{1}{2}\right), \\
& \alpha(0,0)=\frac{1}{\sqrt{2}}\left[\varphi_{1}\left(\frac{1}{2}, \frac{1}{2}\right) \varphi_{2}\left(\frac{1}{2},-\frac{1}{2}\right)-\varphi_{2}\left(\frac{1}{2}, \frac{1}{2}\right) \varphi_{1}\left(\frac{1}{2},-\frac{1}{2}\right)\right],
\end{aligned}
$$

where the first three form a spin triplet $\left({ }^{3} S_{1}\right)$ of $S=1$ and $S_{z}=1,0,-1$ states have the property that they are symmetric under particle interchange, and the last one is a singlet $\left({ }^{1} S_{0}\right)$ of $S=S_{z}=0$ state has the property of antisymmetric. The symmetry of the spin function $\alpha$ under particle interchange is $(-1)^{S+1}$, where $S$ is the total spin. Particle interchange is equivalent to space inversion, introducing a factor $(-1)^{l}$, where $l$ is the orbital angular momentum of the system. The charge wave function $\beta$ acquire a charge conjugation factor $C=(-1)^{n}$ where $n$ is the number of photons under particle interchange. The product of the factors applying to the separate spin, space, and charge function must then be that of the total wave function $\varphi$, which we denote by $K$, so that $K=C(-1)^{S+1}(-1)^{l}$. Individuality of the singlet and the triplet states Ps are presented in 
Table 1.

\subsection{Comparison of Exotic and Non Exotic Atoms}

Since most of the previous studies have focused on the Galactic centre as the most promising source of Ps, although visual extinction is very high. However, detection of Ps recombination line made by Puxley \& Skimer (1996) who searched for Ps Paschen- $\beta$ from the Galactic centre but undetected [23]. Very recently study chromaticity of solar spectrum lead me to discover the solar Ps which is described in the following sections. This renewed interest is motivated by several recent and imminent advances in technology. From the literature survey it is found that Ps formation, recombination and quenching largely take place at temperature $<10^{6} \mathrm{~K}$. Therefore, most astrophysical environments $\left(\sim 10^{3}-10^{6} \mathrm{~K}\right)$ for Ps formation will be the dominant process leading to annihilation [24]. A comparative study of Ps, $\mathrm{H}$ and He are shown in Table 2.

The spectral lines (wavelength) of solar radiation spectrum are achieved from the Rydberg-Ritz formula:

$$
\lambda=\left(\frac{h c}{R_{\alpha}}\right)\left(\frac{n_{i}^{2} n_{f}^{2}}{n_{f}^{2}-n_{i}^{2}}\right)
$$

where $h$ is the Planck's constant, $c$ is the speed of light, $R_{\alpha}$ is the Rydberg energy (shown in Table 2). Hence, wavelength of each atom will be different i.e., different color bands in the spectrum. The quantum numbers $n_{i}$ and $n_{f}$ respectively are the initial and final states of the atomic transition. For the Lyman series $n_{i}=1$ and $n_{f}=2,3,4, \cdots$; for the Balmer series $n_{i}=2$ and $n_{f}=3,4,5, \cdots$; for the Paschen series $n_{i}=3$ and $n_{f}=4,5,6, \cdots$; and for the Brackett series $n_{i}=4$ and $n_{f}=5,6,7, \cdots, \ldots$ etc. The intensity of each color bands will be distributed according to the Planck's radiation formula which is given below and details of this formulation can be found elsewhere [25].

$$
\frac{\mathrm{d} R(\lambda)}{\mathrm{d} \lambda}=\frac{2 \pi h c^{2}}{\lambda^{5}} \frac{1}{\mathrm{e}^{\frac{h c}{\lambda K T}}-1}
$$

where $K$ is the Boltzmann constant and $T$ is the temperature of the photosphere (5800 K). The wavelength $\lambda$ of the corresponding $\mathrm{H}$, He and Ps can be obtained from Equation (1).

\section{Detector Development and Data Acquisition}

In order to study the solar Ps an advanced Solar Spectrum Monitor (SSM) development is extremely vital. Using the various colored LEDs (keeping in mind of VIBGYOR in the solar spectrum Red, Orange, Yellow, Green and White LEDs are taken into account) and a Freeduino the SSM detector is constructed. LEDs which are commer-

\begin{tabular}{|c|c|c|c|c|c|}
\hline \multirow{2}{*}{ Ps states } & \multicolumn{5}{|c|}{ Particle-antiparticle interactions } \\
\hline & $S=J$ & 1 & C & $K$ & Lifetimes (s) \\
\hline Singlet $\left({ }^{1} S_{0}\right) \quad 2 \gamma \quad$ (antisymmetric) & 0 & 0 & +1 & -1 & $1.25 \times 10^{-10}$ \\
\hline Triplet $\left({ }^{3} S_{1}\right) \quad 3 \gamma \quad$ (symmetric) & 1 & 0 & -1 & -1 & $1.42 \times 10^{-7}$ \\
\hline
\end{tabular}

Table 1. Properties of singlet and triplet states Ps.

\begin{tabular}{|c|c|c|c|c|c|c|}
\hline \multirow[b]{2}{*}{ Atoms } & \multicolumn{6}{|c|}{ Properties } \\
\hline & Ground state & $\begin{array}{l}\text { Mass energy } \\
(\mathrm{MeV})\end{array}$ & $\begin{array}{l}\text { Rydberg energy } \\
(\mathrm{eV})\end{array}$ & Lyman- $\alpha$ (nm) & $\begin{array}{l}\text { 1S-2P transition } \\
\text { energy }(\mathrm{eV})\end{array}$ & Stability \\
\hline $\operatorname{Ps}\left(\mathrm{e}^{+} \mathrm{e}^{-}\right)$ & & 1.02 & -6.8 & 243.0 & 5.1 & Unstable (no nucleus) \\
\hline $\mathrm{H}(p, \mathrm{e})$ & & 938.00 & -13.6 & 121.5 & 10.2 & Stable (nucleus exist) \\
\hline $\mathrm{He}(2 p, 2 n, 2 \mathrm{e})$ & & 3756.00 & -54.6 & 58.4 & 21.2 & $\begin{array}{c}\text { Stable \& inert (nucleus } \\
\text { exist) }\end{array}$ \\
\hline
\end{tabular}

Table 2. Comparison among the Ps, $\mathrm{H}$ and He atoms. 
cially available in the public market are collected, hence manufacture's quote of each colored wavelength of LEDs are unavailable. Freeduino is a tool for the interfacing of data to a computer from the physical parameters, e.g., sound, light etc. It is based on the Atmel's ATMEGA328 micro controller systems. The size, shape and number of each colored LEDs are kept equal and connected in series to each group. The size of the Vero-board is $15 \times 10 \mathrm{~cm}^{2}$ and each colored section is about $4 \times 4 \mathrm{~cm}^{2}$. There are five channels of the color group. The analogue output signals from the SSM are fed into the Freeduino digital microprocessor unit 0 - 4. The Freeduino gets its input i.e., analogue signals from the $S S M$ detector when it is directly exposed to the ray of Sun for an hour. These signals are digitized by the $A D C$ (1024 Channels) of the board that is interfaced with a PC via USB ports and output data is scrutinized in the monitor by using external software. This data is now displayed and stored in the ASCII format for further analysis. In order to take the background data SSM detector is covered with a piece of thick black cloth and data are taken for the same time. A schematic diagram of the SSM detector is shown in Figure 1.

\section{Results and Discussions}

Data obtained by different colored $L E D s$ are compared with the results of the simulation considering the model based on Rydberg-Ritz-Planck principle rigorously.

\subsection{Simulation}

In order to understand the solar radiation spectrum intensively, an extensive simulation is carried out for the atomic transitions of He, Ps and $\mathrm{H}$ according to the Rydberg-Ritz principle (see Equation (1)). Simulation results are shown in Figures 2(a)-(c) and solar spectrum (sum of $\mathbf{a}, \mathbf{b}$ and $\mathbf{c}$ ) is depicted in (d) where components of each atomic transitions are clearly exhibited with the colour bands, but data do not illustrate the distribution of solar radiation spectrum.

Hence wavelengths which are obtained by the Rydberg-Ritz's formula stated in Equation (1) are fed into the Planck's radiation formula. The wavelength distributions are shown in Figures 3(a)-(c). After combining those data of Ps, He and $\mathrm{H}$ the solar radiation spectrum is achieved and that is revealed in (d) and consequently the area of interest (720 - $830 \mathrm{~nm}$, Ps-Balmer series) is illuminated.

Theoretical contribution of H-(Balmer+Paschen), He-(Paschen+Brackett) and Ps-(Balmer) spectra in visible range of solar radiation respectively are estimated to be $37 \%, 41 \%$ and $22 \%$.

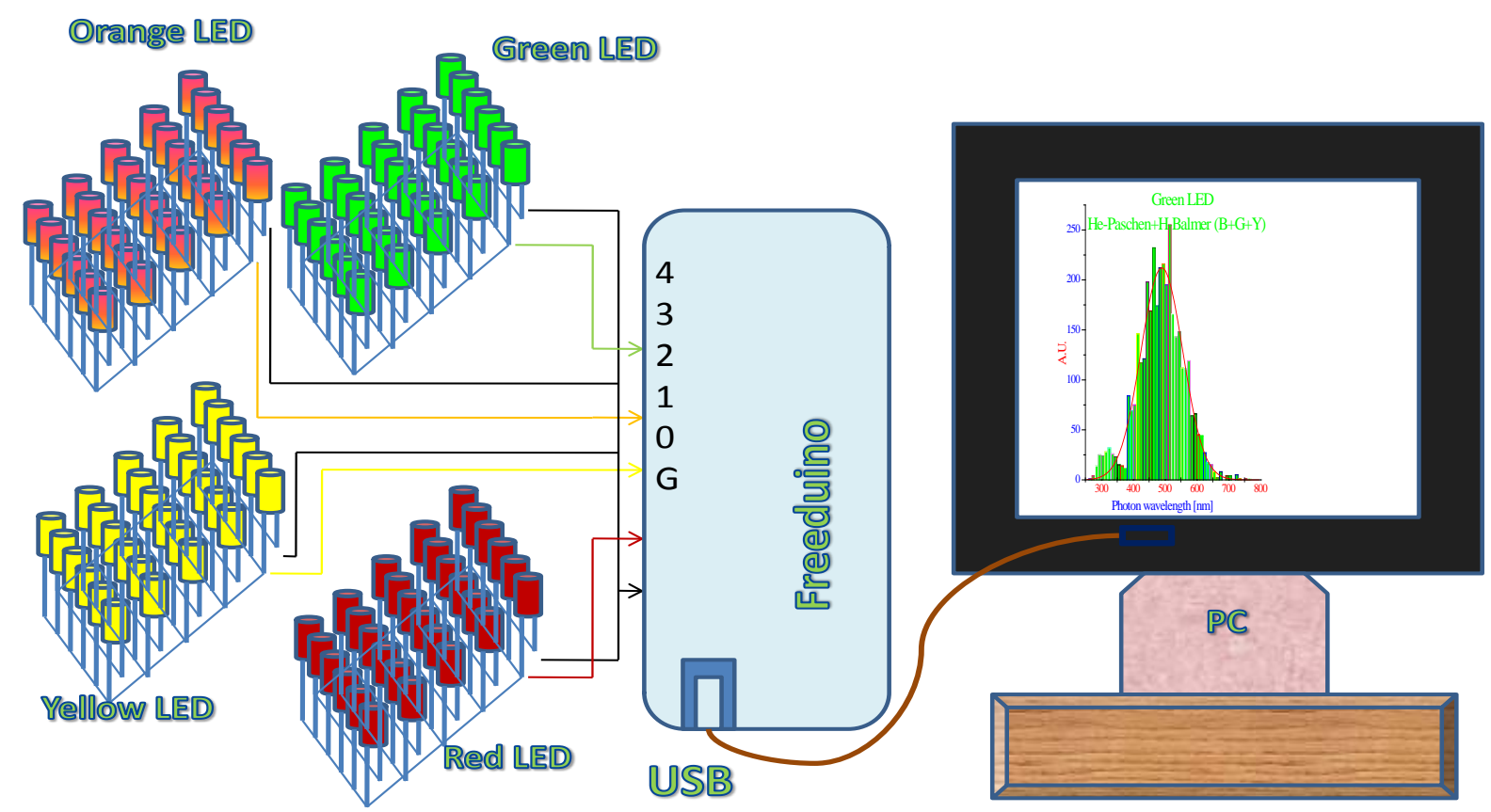

Figure 1. Experimental arrangement of solar spectrum monitor. 

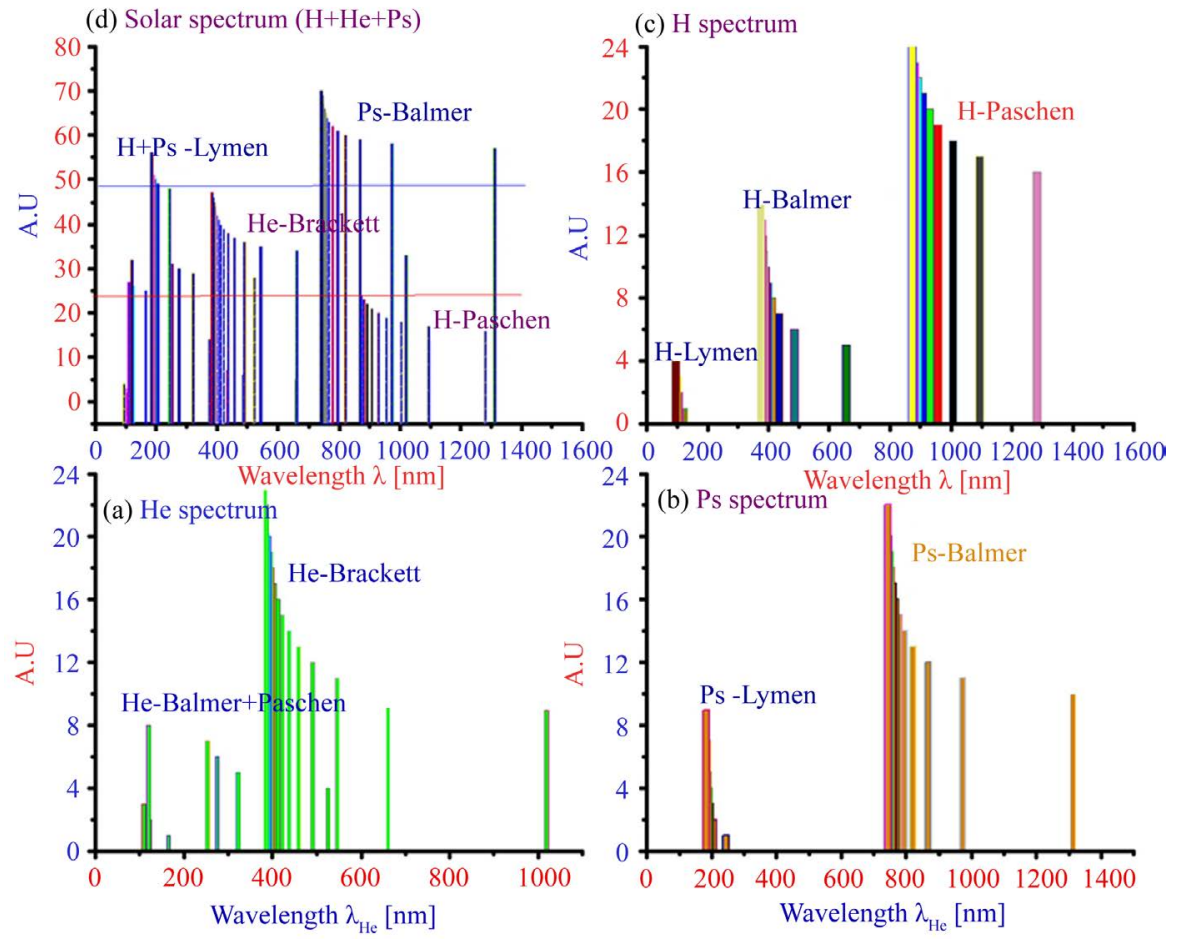

Figure 2. Origin of the solar spectrum.

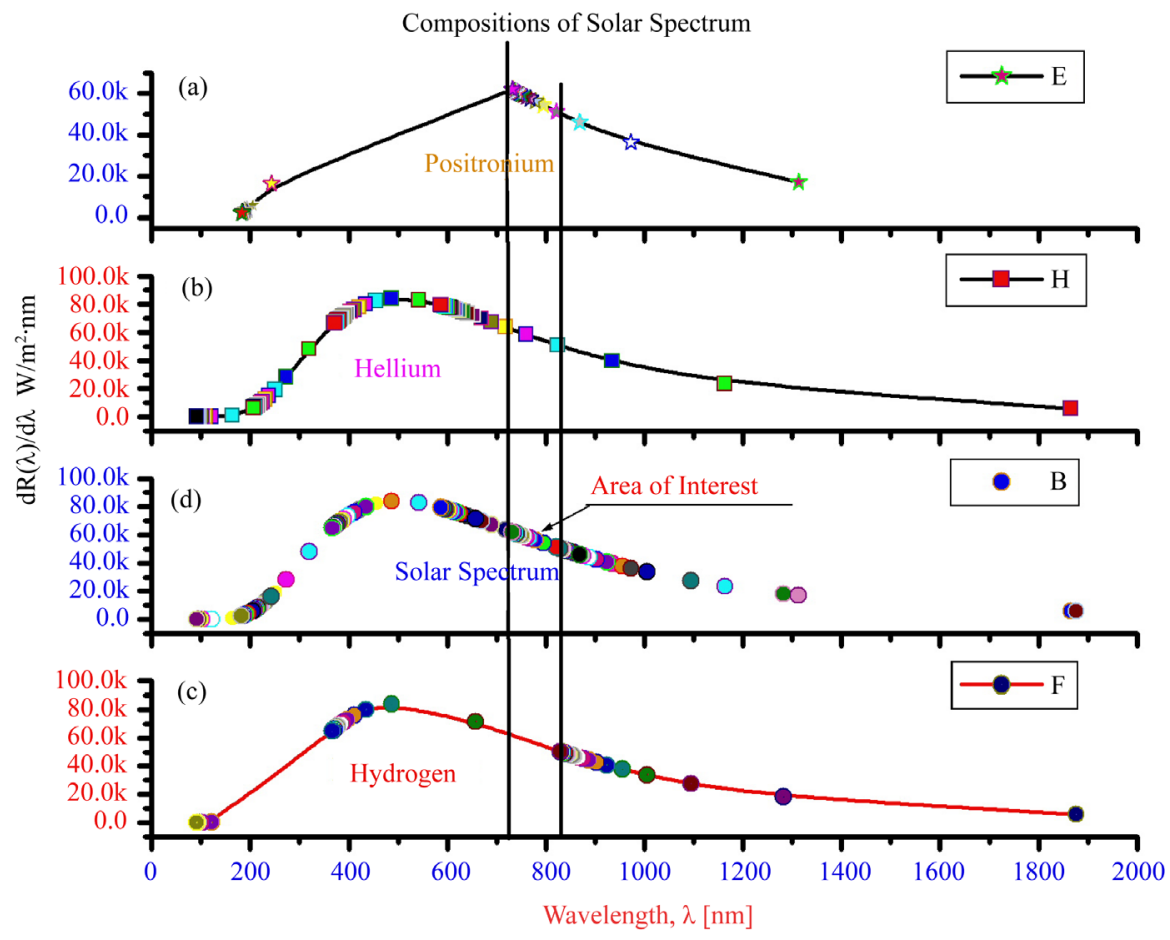

Figure 3. Distribution of solar radiation with other constituent spectra.

\subsection{Experimental Results}

Similarly experimental data are analyzed by subtracting each background from the respective colored bands and converted energy $(\mathrm{eV})$ to wavelength $(\mathrm{nm})$ which is depicted in Figures 4(a)-(d). In order to see the contribu- 

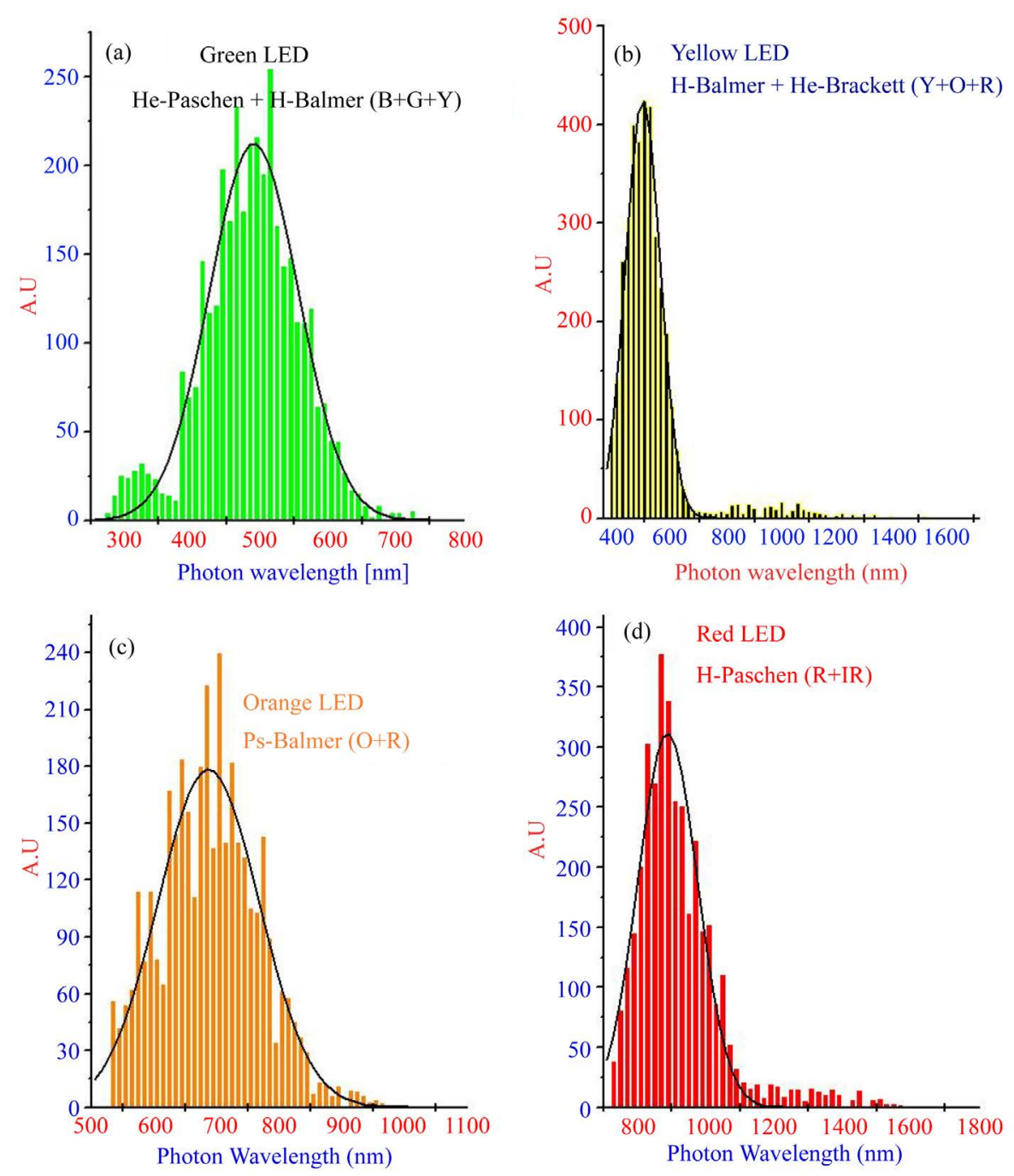

Figure 4. Observation of solar radiation by coloured $L E D$ s.

tion of each component in the solar radiation spectrum data are fitted with Gaussian. The mean and FWHM $(=2.35 \times \sigma)$ of the Gaussian are shown in Table 3 and considered for the evaluation with a $L E D$ manufacturer's quoted values found elsewhere [26] for better understanding.

Contribution of H-Balmer 54\% in Green, H-Paschen 4.2\% in Orange and 66\% in Red spectra are estimated from the fitted frequencies. Similarly He-Paschen 8\% in Green, 2.34\% in Yellow, and He-Brackett 34\% in Green, 93\% in Yellow; and Ps-Balmer 1\% in Yellow, 41\% in Orange and 26\% in Red are estimated. In total contributions of H-(Balmer + Paschen), He-(Paschen + Brackett) and Ps-(Balmer) respectively are determined to be $31 \%, 52 \%$ and $17 \%$ in the visible range of solar radiation spectrum.

The extremity at right hand side of each spectrum shows the temperament of Planck's radiation formula. In order to find exact distribution of this formula more colored LEDs (Violet, Indigo and Blue) data are required. However reported results confirm a good agreement with the theoretical achievement of Ridberg-Ritz-Planck's principle within this constraint. The differences what are merely appeared due to the diverse transitional times among $\mathrm{H}, \mathrm{He}$ and Ps in the solar environment, refraction and reflection of light due to solar and earth atmospheres and statistical error due to electronics fluctuations which are not determinative. The FWHM of each Gaussian distribution represents the wide range of colors originated from the respective atoms of different transition series. The FWHM of Red spectrum shows maximum due to the widest range of different series and the highest contribution of H-Paschen (66\%) series. Yellow spectrum shows minimum because of the shorter range and maximum contribution of He-Brackett (93\%) series. The contribution of the He atom is maximum because 
Table 3. Evaluation of experimental results with manufacturer's quoted value.

\begin{tabular}{|c|c|c|c|c|}
\hline \multirow{3}{*}{$\begin{array}{l}\text { Chemical composition of } \\
\text { LEDs }\end{array}$} & \multicolumn{4}{|c|}{ Wavelengths (nm) } \\
\hline & \multicolumn{2}{|c|}{ Manufacturer's quoted values ${ }^{\mathrm{a}}$} & \multicolumn{2}{|c|}{ Experimental values } \\
\hline & Mean wavelength & FWHM & Mean wavelength & FWHM \\
\hline GaP-GaP & 555 (Green) & 25 & 490 (Green) & 154 \\
\hline GaAsP-GaP & 610 (Orange) & 35 & 492 (Yellow) & 150 \\
\hline GaAlAs-GaAlAs & 660 (Red) & 25 & 687 (Orange) & 190 \\
\hline GaAlAs-GaAs & 700 (Red) & 25 & & \\
\hline GaAlAs-GaAlP & 880 (Near IR) & 80 & 887 (Red) & 205 \\
\hline GaAs-GaAs & 940 (IR) & 45 & & \\
\hline
\end{tabular}

a. Reference [26] is not the same manufacture of LEDs which are used in this experiment.

it is stable and inert. On the other hand, higher ionization probability leads $\mathrm{H}$ for taking part in the fusion reaction. Ps is unstable, quenching in the huge magnetic field and have the shortest lifetimes; as a result transitional probabilities of $P$ s are fewer than $\mathrm{H}$ and He. In the Orange spectrum (600 - $700 \mathrm{~nm}$ ), around $40 \%$ contribution comes from the He-Pfund series. But I suppose this probability is very low in compare to He-Brackett and He-Paschen series in the range of visible light. Hence, it is better to think other type of lighter atoms forming in the fusion mechanisms whose binding energy $(\sim 8.25 \mathrm{eV})$ is fewer than $\mathrm{H}$ and greater than Ps atom. That hypothetical atom (presume Nm) will provide the color band of wavelength $(600-700 \mathrm{~nm})$ from the transition of Nm-Balmer series.

\section{Conclusion}

The data of Solar Spectrum Monitor conclude the existence of solar Ps which is one of the main constituent of solar radiation spectrum. By monitoring and characterizing the colored radiation spectra solar Ps will be estimated and that will tell us the present, past and future of the astrophysical phenomena. If SSM detector is possible to send in the outer space with necessary modification and collect the $\gamma$-rays of $\mathrm{e}^{+} \mathrm{e}^{-}$annihilation more precisely and those analyzed data will provide intense information about the space, extra galactic solar system and their circumstances, celestial Ps, dark matter and possibly the giant source of PsBEC. Hence, solar Ps spectroscopy plays a crucial role for the determination of sun's environment, fusion reaction in various cells and constituents. The intensity distribution of the solar spectrum and associate color bands exactly tell us the nuclear and fusion reaction mechanisms, radiation energy and particle-antiparticle plasma in the colossal magnetic fields.

\section{Acknowledgements}

I profoundly acknowledge the financial support and cooperation of my family for executing this experiment and publishing the research paper in the IJAA.

\section{References}

[1] Mondal, N.N. (2014) Study Chromaticity of Solar Spectrum. International Journal of Astronomy and Astrophysics, 4, 510-518. http://dx.doi.org/10.4236/ijaa.2014.43047

[2] Berko, S. and Pendleton, H.N. (1980) Positronium. Annual Review of Nuclear and Particle Science, 30, 543-581. http://dx.doi.org/10.1146/annurev.ns.30.120180.002551

[3] Anderson, C.D. (1933) Positrons from Gamma-Rays. Physical Review, 43, 491. http://dx.doi.org/10.1103/PhysRev.43.1034 http://dx.doi.org/10.1103/PhysRev.43.368 http://dx.doi.org/10.1103/PhysRev.43.491

[4] Canter, K.F., Mills Jr., A.P. and Berko, S. (1975) Observation of Positronium Lyman- $\alpha$ Radiation. Physical Review Letters, 34, 177. http://dx.doi.org/10.1103/PhysRevLett.34.848.2 http://dx.doi.org/10.1103/PhysRevLett.34.177

[5] Mondal, N.N., Hirose, T., Hamatsu, R., Kumita, T., Ijima, H., Irako, M. and Omori, T. (1999) Construction of a Time- 
of-Flight Measurement System to Study the Low Energy Positronium Production. Applied Surface Science, 149, 269275. http://dx.doi.org/10.1016/S0169-4332(99)00214-7

[6] Mondal, N.N. (2008) A New Approach to Position Reconstruction in TOFPET. Journal of Medical Physics, 33, 9-13. http://dx.doi.org/10.4103/0971-6203.39418 http://www.jmp.org.in/text.asp?2008/33/1/9/39418

[7] Mondal, N.N. (2009) Monte Carlo Simulation Studies on Scintillation Detectors and Image Reconstruction of BrainPhantom Tumors in TOFPET. Journal of Medical Physics, 34, 212-216. http://dx.doi.org/10.4103/0971-6203.56083 http://www.jmp.org.in/text.asp?2009/34/4/212/56083

[8] Coleman, P.G. (2001) Positron Depth Profiling. Materials Science Forum, 363-365, 420-424. http://dx.doi.org/10.4028/www.scientific.net/MSF.363-365.420

[9] Krause-Rehberg, R., Borner, F. and Redman, F. (2001) Positron Beam Studies of Defects in Semiconductors. Materials Science Forum, 363-365, 404-408. http://dx.doi.org/10.4028/www.scientific.net/MSF.363-365.404

[10] Ganguly, B.N., Mondal, N.N., Nandi, M. and Roesch, F. (2009) Some Physical Aspects of Positron Annihilation Tomography: A Critical Review. Journal of Radioanalytical and Nuclear Chemistry, 279, 685-698. http://dx.doi.org/10.1007/s10967-007-7256-2

[11] Cassidy, D.B. and Mills Jr., A.P. (2007) The Production of Molecular Positronium. Nature, 416, 345.

[12] Cassidy, D.B., Hisakado, T.H., Tom, H.W.K. and Mills Jr., A.P. (2011) Photoemission of Positronium from Si. Physical Review Letters, 107, Article ID: 033401. http://dx.doi.org/10.1103/PhysRevLett.107.033401

[13] Mondal, N.N. (2002) A Novel Detection System for Laser Cooling of Ortho-Positronium. Nuclear Instruments and Methods in Physics Research Section A, 495, 161-169. http://dx.doi.org/10.1016/S0168-9002(02)01578-4

[14] Chu, S. and Mills Jr., A.P. (1982) Excitation of the Positronium 1 S13 $\rightarrow 2$ S13 Two-Photon Transition. Physical Review Letters, 48, 1333-1337. http://dx.doi.org/10.1103/PhysRevLett.48.1333

[15] Nagashima, Y., Hakodate, T., Miyamoto, A. and Michishio, K. (2008) Efficient Emission of Positronium Negative Ions from Cs Deposited W (100) Surfaces. New Journal of Physics, 10, Article ID: 123029. http://dx.doi.org/10.1088/1367-2630/10/12/123029

[16] Cassidy, D.B., Hisakado, T.H., Tom, H.W.K. and Mills Jr., A.P. (2012) Optical Spectroscopy of Molecular Positronium. Physical Review Letters, 108, Article ID: 133402. http://dx.doi.org/10.1103/PhysRevLett.108.133402

[17] Cassidy D.B., Hisakado, T.H., Tom, H.W.K. and Mills Jr., A.P. (2012) Efficient Production of Rydberg Positronium. Physical Review Letters, 108, Article ID: 043401. http://dx.doi.org/10.1103/PhysRevLett.108.043401

[18] Chupp, E.L., Forrest, D.J., Higbie, P.R., Suri, A.N., Tsai, C. and Dunphy, P.P. (1973) Solar Gamma Ray Lines Observed during the Solar Activity of August 2 to August 11, 1972. Nature, 241, 333-335. http://dx.doi.org/10.1038/241333a0

[19] Leventhal, M., MacVallum, C.J. and Stang, P.D. (1978) Detection of $511 \mathrm{keV}$ Positron Annihilation Radiation from the Galactic Center Direction. Astrophysical Journal, 225, L11-L14.

[20] Gould, R.J., (1989) Direct Positron Annihilation and Positronium Formation in Thermal Plasmas. Astrophysical Journal, 344, 232. http://dx.doi.org/10.1086/167792

[21] Canter, K.F., Mills Jr., A.P. and Berko, S. (1974) Efficient Positronium Formation by Slow Positrons Incident on Solid Targets. Physical Review Letters, 33, 7-10. http://dx.doi.org/10.1103/PhysRevLett.33.7

[22] Perkins, D.H. (1986) Introduction to High Energy Physics. Addison-Wesley Publishing Company, Inc., Tokyo.

[23] Puxley, P.J. and Skinner, C.K. (1996) A Search for Infrared Positronium Line Emission from the Great Annihilator near the Galactic Centre. Proceedings of the Galactic Center, 4th ESO/CTIO Workshop, La Serena, 10-15 March 1996, 439.

[24] Ellis, S.C. and Hawthorn, B. (2009) The Search for Celestial Positronium via the Recombination Spectrum. The Astrophysical Journal, 707, 457-471. http://dx.doi.org/10.1088/0004-637X/707/1/457

[25] Rohlf, J.W. (1994) Modern Physics from $\alpha$ to Zo. John Wiley \& Sons Inc., Hoboken.

[26] Mims III, F.M. (1992) Sun Photometer with Light-Emitting Diodes as Spectrally Selective Detectors. Applied Optics, 31, 6965-6967. http://dx.doi.org/10.1364/AO.31.006965 
Scientific Research Publishing (SCIRP) is one of the largest Open Access journal publishers. It is currently publishing more than 200 open access, online, peer-reviewed journals covering a wide range of academic disciplines. SCIRP serves the worldwide academic communities and contributes to the progress and application of science with its publication.

Other selected journals from SCIRP are listed as below. Submit your manuscript to us via either submit@scirp.org or Online Submission Portal.
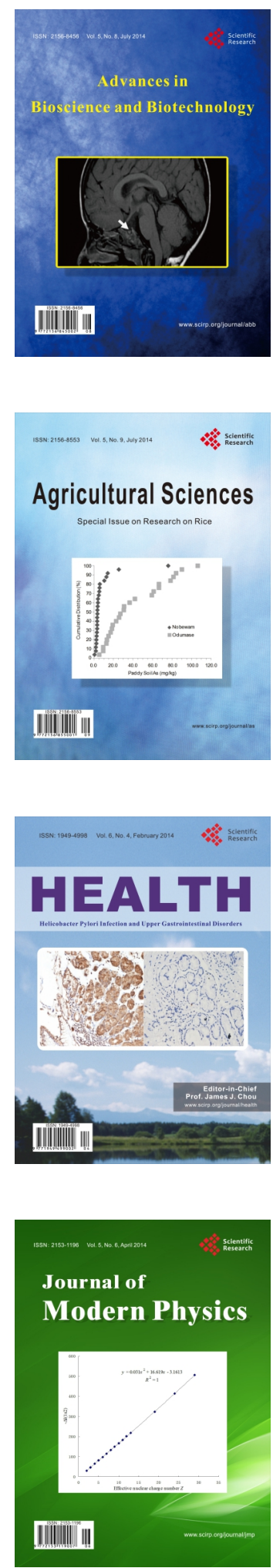
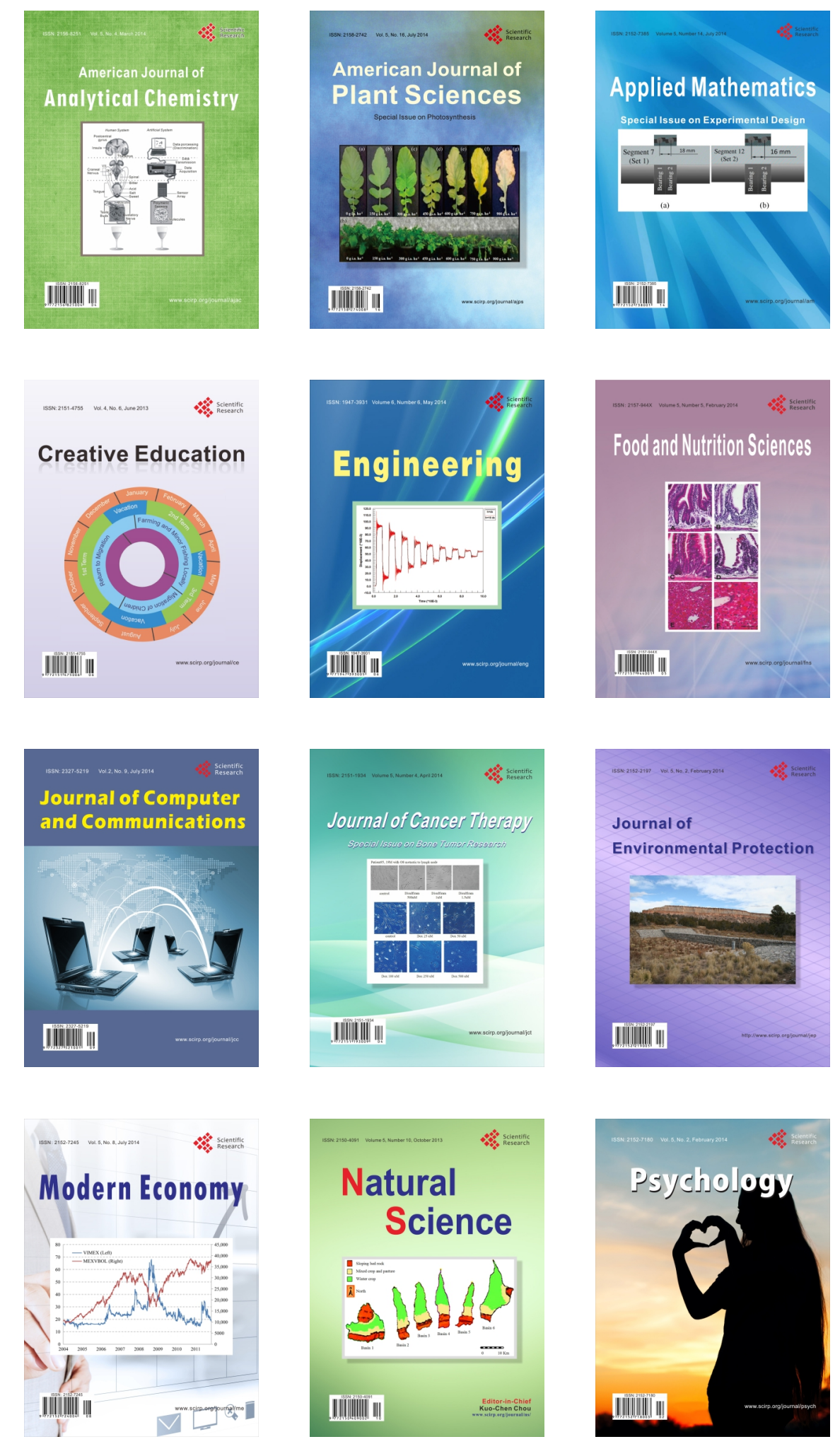